\title{
Tirofiban combined with urokinase selective intra-arterial thrombolysis for the treatment of middle cerebral artery occlusion
}

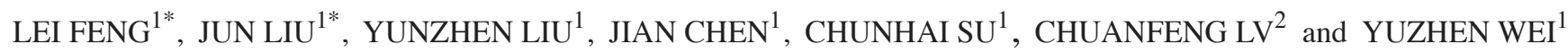 \\ Departments of ${ }^{1}$ Neurovascular Surgery and ${ }^{2}$ Clinical Pharmacy, Jining No. 1 People's Hospital, \\ Jining, Shandong 272111, P.R. China
}

Received August 22, 2014; Accepted June 24, 2015

DOI: $10.3892 /$ etm.2016.2995

\begin{abstract}
The aims of the present study were to establish a model of embolic stroke in rabbits and to evaluate the efficacy and safety of intra-arterially administered tirofiban combined with urokinase thrombolysis. The middle cerebral artery occlusion model (MCAO) of embolic stroke was established in New Zealand rabbits via an autologous clot. The model rabbits were allocated at random into four groups: Tirofiban group (T group), urokinase group (UK group), tirofiban and urokinase group ( $\mathrm{T}+\mathrm{UK}$ group) and the control group (C group). The recanalization rate, relative-apparent diffusion coefficient (rADC) and neurological function deficit score (NFDS) values were compared among the four groups. The recanalization rate, rADC and NFDS values were improved in the T + UK group compared with the other groups. In summary, the intra-arterial administration of tirofiban combined with urokinase thrombolysis was a more effective intervention in an MCAO model compared with intra-arterial urokinase alone, and may promote reperfusion and reduce infarct volume.
\end{abstract}

\section{Introduction}

Thrombolysis is an approved, effective treatment for acute ischemic stroke if administered within the first $4.5 \mathrm{~h}$ after the onset of symptoms; however, thrombolysis increases the risk of symptomatic intracranial hemorrhage, which occurs in $5-10 \%$ of patients (1-4). In addition, due to the limits of the thrombolysis time window, $<10 \%$ of patients with acute ischemic stroke receive recombinant plasminogen activator (rt-PA) thrombolytic therapy, particularly in developing countries $(5,6)$. Furthermore, the combined thrombolytic

Correspondence to: Dr Lei Feng, Department of Neurovascular Surgery, Jining No. 1 People's Hospital, 6 Jiankang Road, Jining, Shandong 272111, P.R. China

E-mail: leifengcn@126.com

*Contributed equally

Key words: platelet glycoprotein IIb-IIIa complex, thrombolysis, intracranial embolism, rabbit model recanalization rate of the cerebral artery and veins remains insufficient (7). Therefore, there is an urgent requirement for a novel therapy that is able to extend the treatment time window for stroke and decrease bleeding complications.

Recently, experimental studies that investigated the efficacy of a platelet glycoprotein IIb/IIIa receptor antagonist in the treatment of stroke, demonstrated that the treatment was able to reduce the cerebral infarct volume even if it was not administered immediately $(8,9)$. Tirofiban is a non-peptide platelet IIb/IIIa receptor antagonist, and is able to dose-dependently inhibit the platelet aggregation induced by various stimuli and eliminate newly formed thrombus, including thrombus containing fibrin (10). Thus, tirofiban has been frequently used in endovascular interventional therapy to prevent the occurrence of vascular endothelial damage-induced thromboembolic complications and early re-occlusion $(11,12)$.

The aims of the present study were to investigate the feasibility, safety and efficacy of tirofiban, alone and in combination with urokinase, in the treatment of intra-arterial thrombolysis, and to provide a reference for the clinical treatment of acute ischemic stroke.

\section{Materials and methods}

Thrombosis preparation. A total of 40 male New Zealand white rabbits (Wuhan Wanqianjiahe Experimental Animal Breeding Co., Ltd., Wuhan, China), weighing 2.5-3.0 kg, were obtained for the present study. This study was conducted in strict accordance with the recommendations of the Guide for the Care and Use of Laboratory Animals of the National Institutes of Health (1993). The animal use protocol has been reviewed and approved by the Institutional Animal Care and Use Committee of Jining No. 1 People's Hospital (Jining, China).

Rabbits were anesthetized via an intravenous injection of 3\% pentobarbital (Shanghai Research Biological Technology Co., Ltd., Shanghai, China) through the ear vein. Then the rabbit was fixed in place, and the bilateral ears' dorsal surface was disinfected with iodine. An improved lumbar puncture needle (Changzhou Shixing Medical Equipment Co., Ltd., Changzhou, China) was used to puncture and scrape the artery intima of bilateral ears. After $\sim 2 \mathrm{~cm}$ of the artery intima had been abraded, the proximal end of the rabbit ear artery was ligated with a suture to reduce the blood flow velocity 
and increase the possibility of embolus formation. Rabbits were subsequently returned to the cage and fed. After $24 \mathrm{~h}$, the rabbit was re-anesthetized and fixed in place in order to remove the scraped ear artery. The intravascular thrombus was then removed under magnification. Following extraction, the thrombus was cut into $0.5 \times 0.4-\mathrm{mm}$ pieces using an ophthalmic scalpel and stored in sterile saline for future use.

Establishment of the embolism model. Rabbits that had been subjected to ear artery abrasion and thrombosis removal were fixed supinely on the operating table and routinely disinfected. The interior skin of the right femur was incised, the right femoral artery was separated, hung with a thin thread and prepared for intubation and thrombolysis. The distal end of the right femoral artery was ligated, and the proximal end was temporarily occluded using an arterial clip. Next, half of the right femoral artery was cut at a $45^{\circ}$ angle using ophthalmic scissors. A $4 \mathrm{~F}$ sheath catheter was placed into the right femoral artery, followed by an Echelon-10 microcatheter (ev3 Neurovascular, Irvine, CA, USA) under TV guidance. Under road-map guidance, the right or left common carotid artery was selected for insertion; the catheter tip reached approximately the lateral side of the inferior edge of the second cervical vertebrae and was set as the pathway. At this site, the internal carotid artery was visible as a branch of the common carotid artery, which expanded backwards and upwards, and with an ampulla-like swelling at the internal carotid artery as a marker. The catheter was inserted into the proximal end of the internal carotid artery, passed the occipital artery and created an opening for normal and lateral angiography to determine the dilation of the blood vessels. After no significant regurgitation was confirmed using a contrast agent bolus administered manually, a 1-ml syringe was used to inject three thrombus strips into the internal carotid artery. The catheter was then withdrawn after the injection of contrast agent had confirmed the occlusion of the middle cerebral artery. Following the embolization, the animals were housed at $\sim 37^{\circ} \mathrm{C}$, and the vascular recanalization was reconfirmed at 2 and $5 \mathrm{~h}$ using angiography. The catheter was flushed with heparin saline throughout the surgery (4).

Grouping and treatment. The successful establishment of a model of embolic stroke in New Zealand white rabbits was confirmed by digital subtraction angiography using an OEC 9800 workstation (GE Healthcare, Milwaukee, WI, USA). The model rabbits were allocated at random into four treatment groups ( $\mathrm{n}=10$ per group): Tirofiban ( $\mathrm{T}$ group), Urokinase (UK group), tirofiban + urokinase group $(\mathrm{T}+\mathrm{UK}$ group) and the control group (C group). Each group was subjected to $1.5 \mathrm{~T}$ magnetic resonance imaging (MRI) T1-weighted imaging (T1WI), T2WI and diffusion (D)WI examination $2 \mathrm{~h}$ following modeling, using a SIGNA high-speed MRI scanner (GE Healthcare).

At $2 \mathrm{~h}$ after infarction, the $\mathrm{T}$ group was slowly infused with $20 \mathrm{ml}$ saline + tirofiban $(5 \mu \mathrm{g} / \mathrm{kg}$; Wuhan Yuancheng Technology Development Co., Ltd., Wuhan, China) using an internal-carotid-artery microcatheter, which was completed within $10 \mathrm{~min}$. The UK group received $20 \mathrm{ml}$ saline + urokinase (25,000 U/kg; Wuhan Yuancheng Technology Development Co., Ltd.) within $10 \mathrm{~min}$. The $\mathrm{T}+\mathrm{UK}$ group was slowly treated with $10 \mathrm{ml}$ saline + urokinase $(15,000 \mathrm{U} / \mathrm{kg})$ within $10 \mathrm{~min}$, followed by $10 \mathrm{ml}$ saline + tirofiban $(3 \mu \mathrm{g} / \mathrm{kg})$ within $10 \mathrm{~min}$. Finally, the $\mathrm{C}$ group was slowly given $20 \mathrm{ml}$ saline.

\section{Detection methods and observation outcomes}

Recanalization rate. The rabbits in each group were bolus-injected with $2 \mathrm{ml}$ iopromide (Ultravist, $300 \mathrm{~g} / \mathrm{l}$, diluted to 150 g/l; Schering Pharmaceutical Co., Ltd., Guangzhou, China) using a high-pressure syringe and an Echelon-10 microcatheter. The microcatheter was detained in the internal carotid artery at $30 \mathrm{~min}$ and $1 \mathrm{~h}$ after the treatment for digital subtraction angiography (pressure, $50 \mathrm{~Pa}$; rate, $0.5 \mathrm{ml} / \mathrm{sec}$ ) to observe the degree of recanalization. Recanalization rate $(\%)=$ Number of rabbits with MCA thrombolytic recanalization/total number of experimental rabbits x 100 .

MRI examination. The animals were immediately subjected to DWI and T2WI examination after $2 \mathrm{~h}$ of successful modeling and $2 \mathrm{~h}$ following the thrombolysis. The SIGNA highspeed MRI scanner was used, with a small joint coil. DWI was performed using the following parameters: Echo planar imaging (EPI) sequence; time of repetition (TR), 5,000 msec; time of echo (TE), $10 \mathrm{msec}$; slice thickness, $3 \mathrm{~mm}$; spacing, $1 \mathrm{~mm}$; field of view (Fov), 16x16 cm; matrix, 128x128; and scan time, $40 \mathrm{sec}$. T2WI parameters were as follows: TR, 2,500 msec; TE, $99 \mathrm{msec}$; slice thickness, $3 \mathrm{~mm}$; spacing, $1 \mathrm{~mm}$; Fov, 24x $24 \mathrm{~cm}$; and matrix, 128x128. Finally, T1WI parameters were as follows: TR, $564 \mathrm{msec}$; TE, $15 \mathrm{msec}$; slice thickness, $3 \mathrm{~mm}$; spacing, $1 \mathrm{~mm}$; Fov, 24x24 cm; and matrix, $128 \times 128 \mathrm{~cm}$. The FuncTool 2000 software package (GE Healthcare) was used to conduct the DWI and T2WI examinations. The apparent diffusion coefficient (ADC) was measured, and the contralateral hemisphere, which was at the corresponding position and not subjected to embolization, was set as the reference. The ratio of these two values was the relative apparent diffusion coefficient (rADC). $\mathrm{rADC}=\mathrm{ADC}$ value of infarct area/ADC value of corresponding normal contralateral area. The rADC values of different groups were compared prior to and following treatment.

Neurological impairment (NI) assessment. Using Bederson's 5-point scale (13), scoring was performed within $24 \mathrm{~h}$ after the animals had recovered from the anesthesia to determine the neurological function deficit score (NFDS). The 5-point scoring criteria were as follows: 0 point, no NI symptom; 1 point, rabbit could not fully extend the contralateral paw; 2 points, circled towards the contralateral side; 3 points, leant towards the contralateral side; and 4 points, was unable to walk spontaneously and lost consciousness. The higher the NFDS score, the more severe the NI.

Pathological examination. The differences in animal behavior were examined $24 \mathrm{~h}$ after the thrombosis. Then, the animals were sacrificed via carotid artery exsanguination. The animals were fixed on a console and the brain tissues from the medulla oblongata were completely transected using olecranon pliers, knife, hacksaw and other tools. The brain was then rapidly removed and frozen at approximately $-20^{\circ} \mathrm{C}$ for $20 \mathrm{~min}$, then separated into $5 \mathrm{~mm}$-thick brain slices and incubated in $2 \%$ 2,3,5-triphenyl-2H-tetrazolium chloride solution at $37^{\circ} \mathrm{C}$ for $30 \mathrm{~min}$. Slices were fixed in phosphate buffer solution-prepared formalin, and the ischemic range was determined. Then, the slices were subjected to hematoxylin-eosin (H\&E) staining and embedded in paraffin for pathological observation under a 
Table I. Recanalization rates among different treatment groups and intergroup comparisons $(\mathrm{n}=10)$.

\begin{tabular}{lcccc}
\hline Group & $\begin{array}{c}\text { Complete } \\
\text { recanalization }\end{array}$ & $\begin{array}{c}\text { Partial recanalization } \\
\text { or non-recanalization }\end{array}$ & Statistical analysis \\
\cline { 4 - 5 } T (1) & 3 & 7 & $(1):(2)$ & P-value \\
T + UK (2) & 8 & 2 & $(1):(3)$ & 0.070 \\
UK (3) & 5 & 5 & $(1):(4)$ & 0.065 \\
C (4) & 0 & 10 & $(2):(3)$ & 0.211 \\
$\chi^{2}$ & $17.753^{\text {a }}$ & & $(2):(4)$ & 0.350 \\
& & & $(3):(4)$ & 0.001 \\
\hline
\end{tabular}

${ }^{\mathrm{a}} \mathrm{P}<0.01$. C, control; T, tirofiban; UK, urokinase.

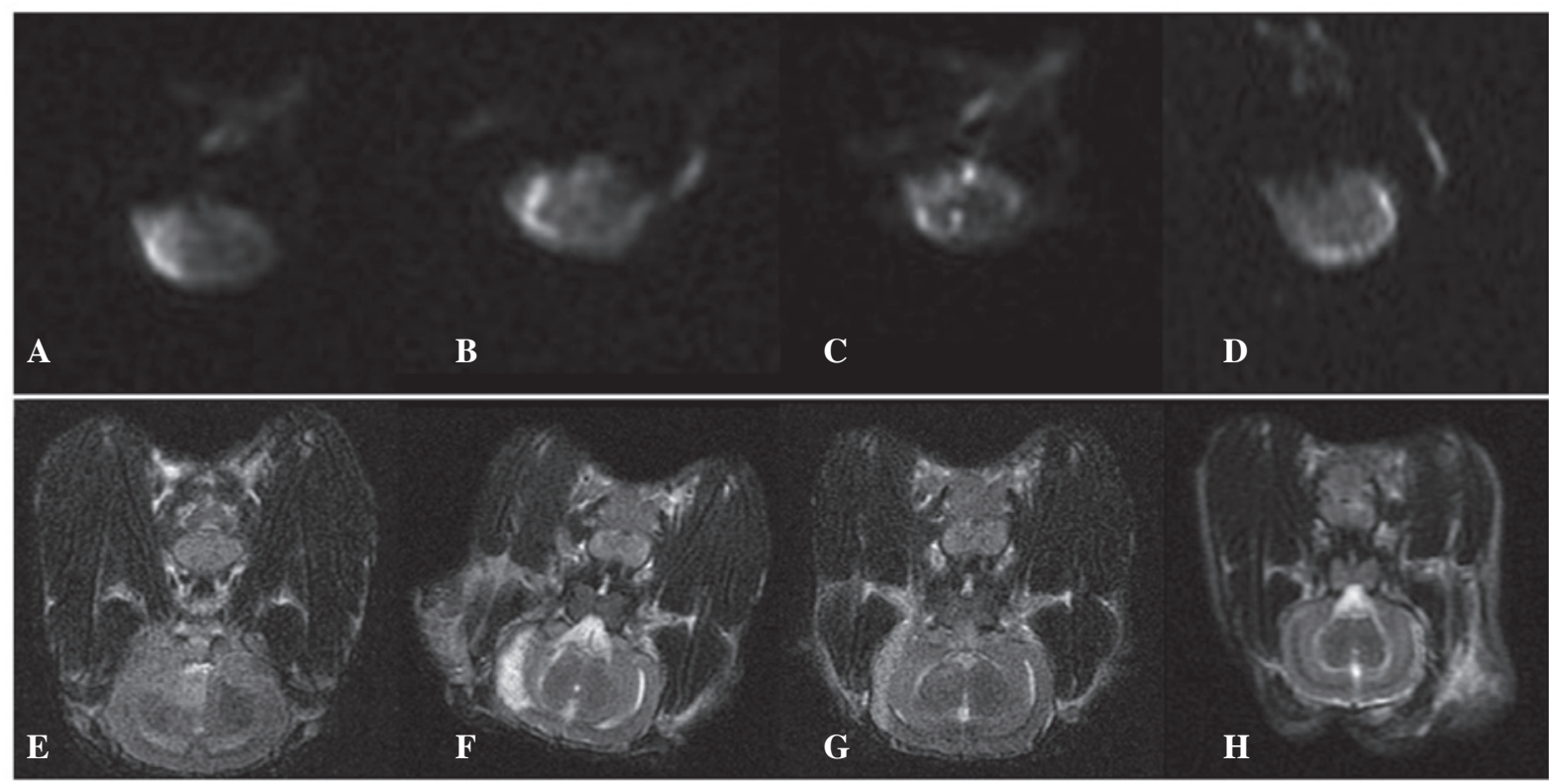

Figure 1. Diffusion-weighted imaging: (A) C group; (B) T group; (C) UK group; (D) T + UK group. T2WI: (E) C group; (F) T group; (G) UK group; (H) T + UK group. C, control; T, tirofiban; UK, urokinase.

light microscope (Olympus BX41; Olympus, Shanghai, China) (Changzhou Shixing Medical Equipment Co., Ltd.). The infarct area was determined using Image-Pro Plus Analysis software (Media Cybernetics, Inc., Rockville, MD, USA).

Statistical processing. SPSS software, version 13.0 was used for data analysis (SPSS Inc., Chicago, IL, USA). Measurement data were expressed as the mean \pm standard deviation. Multi-group comparisons were performed using one-way analysis of variance, and the further pairwise comparison used the least significant difference t-test. Counting data are expressed as ratio (\%), the intergroup comparison used the $\chi^{2}$ test and exact probabilities, with $\mathrm{P}<0.05$ considered to indicate a statistically significant difference.

\section{Results}

Revascularization rate. Statistically significant differences were detected in the recanalization rates among the groups.
The $\mathrm{T}+\mathrm{UK}$ group exhibited the highest revascularization rate, while the $\mathrm{C}$ group presented the lowest rate (Table I).

ADC comparison. Statistically significant differences were detected in the rADC among the four groups. The rADC of the $\mathrm{T}+\mathrm{UK}$ group was the largest, and the rADCs of each group were increased compared with those before the treatment (Fig. 1 and Table II) .

NFDS. The NFDS value of the T + UK group was the lowest among the four groups. Intergroup comparison revealed significant differences between the groups, although not between the T and UK groups, and the T + UK and UK groups (Table III).

Percentage of cerebral infarct area. The T + UK group exhibited the smallest cerebral infarct area, while the $\mathrm{C}$ group presented the largest. Comparisons among the four groups revealed that intergroup differences between pairs of groups 
Table II. rADC of the groups before and after treatment and intergroup comparisons (mean \pm standard deviation, $\mathrm{n}=10$ ).

\begin{tabular}{lcccrr}
\hline Group & & & \multicolumn{2}{c}{ Statistical analysis } & \\
\cline { 4 - 6 } & rADC before & rADC after & Intergroup comparison & P-value & t \\
\hline T (1) & $0.65 \pm 0.03$ & $0.72 \pm 0.06$ & $(1):(2)$ & 0.012 & $3.300^{\mathrm{a}}$ \\
T + UK (2) & $0.67 \pm 0.02$ & $0.88 \pm 0.03$ & $(1):(3)$ & 0.054 & $18.418^{\mathrm{a}}$ \\
UK (3) & $0.65 \pm 0.02$ & $0.75 \pm 0.08$ & $(1):(4)$ & 0.001 & $3.835^{\mathrm{a}}$ \\
C (4) & $0.65 \pm 0.03$ & $0.58 \pm 0.04$ & $(2):(4)$ & 0.021 & $4.427^{\mathrm{a}}$ \\
F & 1.641 & $12.546^{\mathrm{a}}$ & $(3):(4)$ & $<0.001$ & 0.014 \\
\end{tabular}

${ }^{\mathrm{a}} \mathrm{P}<0.01$. rADC, relative apparent diffusion coefficient; $\mathrm{C}$, control; $\mathrm{T}$, tirofiban; UK, urokinase.

were statistically significant $(\mathrm{P}<0.01)$, with the exception of the $\mathrm{T}$ and UK groups (Fig. 2 and Table IV).

$H \& E$ staining. Observation of the $\mathrm{C}$ group under an optical microscope revealed neuronal karyopyknosis, dissolution of nuclear chromatin, marked eosin staining inside the cytoplasm and the degradation of cellular structure. In addition, the $\mathrm{C}$ group exhibited evident brain edema, with numerous neurons exhibiting vacuolar degeneration and mesh-like cells that exhibited severe damage. By contrast, the $\mathrm{T}+\mathrm{UK}$ group exhibited almost normal histology, with a limited number of neurons with vacuolar degeneration that exhibited mild damage. The $\mathrm{T}$ group cells exhibited edema, and numerous cells with vacuolar degeneration exhibited moderate changes. The UK group exhibited limited neuronal degeneration, and necrosis was rare (Fig. 3).

\section{Discussion}

The present study investigated the feasibility, safety and efficacy of combined intra-arterial therapy with low-dose tirofiban and urokinase in a rabbit model of acute ischemic stroke. The early recanalization rate following intravenous rt-PA use is low, with M1 and M2 MCA recanalization rates of $\sim 25$ and 30\%, respectively, for acute cerebral arterial thromboembolism (2) and $4-68 \%$ for occluded macro-vessels $(14,15)$. In a previous study, the average vascular recanalization rate was reported to be $46 \%$ (16), and in another study, the incidence of symptomatic intracerebral hemorrhage was found to be 4-6\% (17). Consistent with the results of the present study, a previous study that used an autologous thrombus to block the MCA revealed that the simple application of a GP IIb/IIIa receptor antagonist was able to induce the recanalization of $33 \%$ of the completely occluded vessels, while a combination of rt-PA (low dose, $10 \mathrm{mg} / \mathrm{kg})$ and high dose thrombolytic therapy $(20 \mathrm{mg} / \mathrm{kg})$ resulted in a recanalization rate of $\sim 66 \%$ (18). Furthermore, Jeon et al (19) observed that the intra-arterial administration of tirofiban during the endovascular treatment of intracranial aneurysms resulted in recanalizations of myocardial infarction (TIMI) grade III in 3 out of 4 patients with total occlusion and 5 out of 6 patients with partial occlusion. The results of the present study indicate that combined intra-arterial therapy with low-dose tirofiban and urokinase reduces MCA throm-
Table III. NFDS comparison among the groups (mean \pm standard deviation, $\mathrm{n}=10$ ).

\begin{tabular}{lccr}
\hline & & \multicolumn{2}{c}{ Statistical analysis } \\
\cline { 3 - 4 } Group & NFDS & Intergroup comparison & P-value \\
\hline T (1) & $1.80 \pm 0.63$ & $(1):(2)$ & 0.009 \\
T + UK (2) & $0.90+0.74$ & $(1):(3)$ & 0.139 \\
UK (3) & $1.40+0.52$ & $(1):(4)$ & $<0.001$ \\
C (4) & $3.20+0.63$ & $(2):(3)$ & 0.098 \\
F & $23.143^{\text {a }}$ & $(2):(4)$ & $<0.001$ \\
& & $(3):(4)$ & $<0.001$ \\
\hline
\end{tabular}

${ }^{\mathrm{a}} \mathrm{P}<0.01$. NFDS, neurological function deficit score; $\mathrm{C}$, control; $\mathrm{T}$, tirofiban; UK, urokinase.

Table IV. Comparison of infarcted area percentage of each group (mean \pm standard deviation, $\mathrm{n}=10$ ).

\begin{tabular}{lccr}
\hline & & \multicolumn{2}{c}{ Statistical analysis } \\
\cline { 3 - 4 } Group & $\begin{array}{c}\text { Infarct } \\
\text { area, \% }\end{array}$ & Intergroup comparison & P-value \\
\hline T (1) & $31.39 \pm 4.75$ & $(1):(2)$ & $<0.001$ \\
T + UK (2) & $20.07+4.03$ & $(1):(3)$ & 0.211 \\
UK (3) & $27.79+5.38$ & $(1):(4)$ & 0.001 \\
C (4) & $38.67+7.03$ & $(2):(3)$ & 0.003 \\
F & $15.241^{\mathrm{a}}$ & $(2):(4)$ & 0.002 \\
& & $(3):(4)$ & 0.001 \\
\hline
\end{tabular}

${ }^{\mathrm{a}} \mathrm{P}<0.01$. C, control; T, tirofiban; UK, urokinase.

bosis and cerebral ischemia without provoking intracranial hemorrhage in a rabbit model of acute ischemic stroke.

The activation, adhesion and aggregation of platelets is a critical factor in the development of arterial thrombosis. The platelet glycoprotein IIb/IIIa receptor is the final common pathway in the induction of platelet aggregation (20). The 

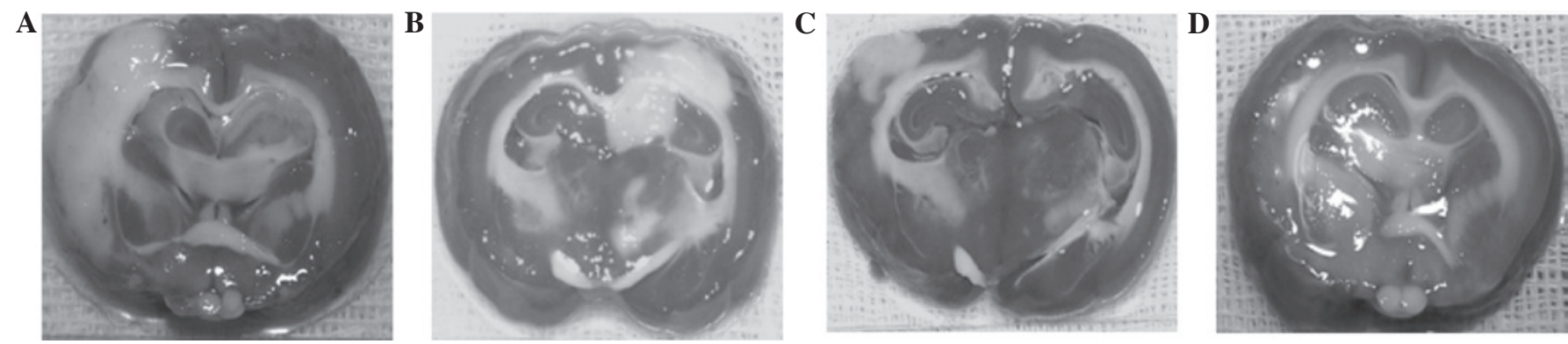

Figure 2. Rabbit brain ischemia modeling was followed by rapid decapitation $24 \mathrm{~h}$ after thrombolysis to extract the brain, 2,3,5-Triphenyl-2H-tetrazolium chloride staining was performed to determine the infarct size. Following staining, the non-ischemic area was rose-red, while the infarct zone was white. (A) Control group; (B) tirofiban group; (C) urokinase group; and (D) tirofiban and urokinase (T + UK) group. The results among the four groups exhibited significant differences, with a significantly reduced cerebral infarct area detected in the $\mathrm{T}+\mathrm{UK}$ group.
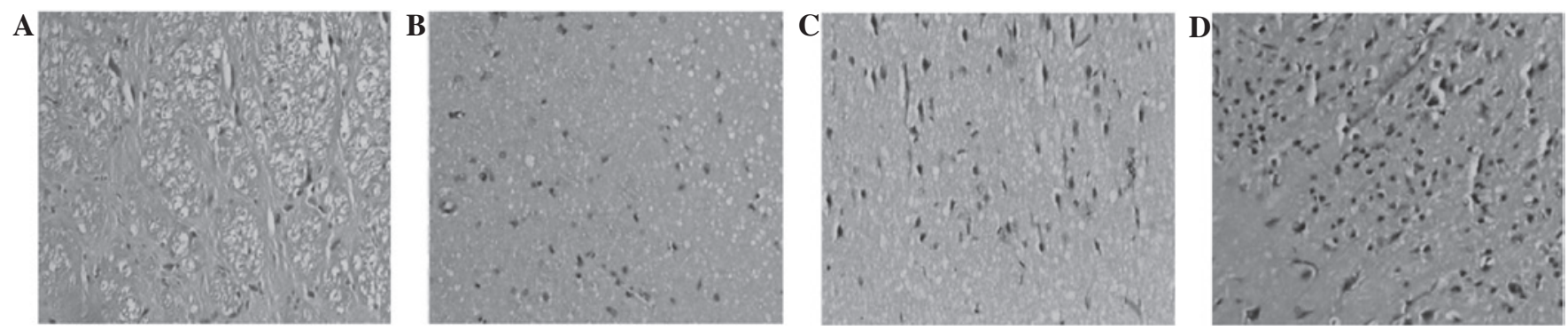

Figure 3. Pathological results of hematoxylin and eosin staining of each group under a light microscope (magnification, $\mathrm{x} 400$ ). (A) In the control group, the neuronal necrosis was extensive. In the (B) tirofiban and (C) urokinase groups, some neuronal structures were normal, while other neurons degenerated. (D) In the tirofiban and urokinase group, the neuronal structures were normal.

combined application of antiplatelet agents enhanced the thrombolytic effects. The thrombolytic therapy restored cerebral blood flow and improved the blood supply towards the ischemic area, while the antiplatelet agent prevented post-thrombolytic restenosis and inhibited the formation of thrombosis in the microcirculatory system. Therefore, the combined application of the two treatments described for the thrombolytic treatment of acute cerebral infarction may be a feasible and effective intervention. The results of the present study indicate that the recanalization rate of tirofiban-urokinase combination in the treatment of acute cerebral infarction was notably increased compared with the application of tirofiban or urokinase alone, and further increased compared with the control group. It has previously been considered that tirofiban functions only as an anticoagulant (21). However, the experimental results of the current study indicate that tirofiban additionally exhibits thrombolytic effects, and that urokinase is able to enhance the thrombolytic efficacy of tirofiban. Thus, tirofiban and urokinase may be co-applied in order to achieve clinical anticoagulation and thrombolysis for the treatment of cerebral infarction. This combination may enhance the anticoagulatory and thrombolytic efficacies of the thrombolytic drugs.

In the present study, rADC was used as an indicator of the extent of the cerebral infarction. Following the combination therapy, the rADC of the T + UK group was elevated compared with the other groups, and the histological observation indicated that the size of the infarct area was significantly reduced. Furthermore, after the drugs were injected into the animals via intracerebral arteries, no new intracerebral hemorrhage was detected. Recently, the application of ultraselective intra-arterial tirofiban thrombolysis has been reported to be safe and effective for the treatment of acute thrombotic events occurring during the treatment procedure of rupture or non-ruptured aneurysm embolization, and the incidence of intracranial hemorrhage was reported to be low (20,22-25). The ultraselective intra-arterial injection of tirofiban may enable the dosage to be reduced while improving the local drug concentration at the thrombotic site and reducing the thrombolysis-induced systemic fibrinolysis and the incidence of intracranial hemorrhage $(10,20,26)$. These results indicate that the combined therapy exhibited good therapeutic value for the recovery of post-embolic brain tissues. As the cerebral intra-arterial injection method was safe and effective, the application of low-dose tirofiban and urokinase may be a feasible intervention. NFSD was used as an effective indicator of neurological recovery in the experimental animals; the lower the NFDS value, the better the functional recovery (27). Observations using light microscopy indicated that the T + UK group exhibited normal histology, cells with neuronal vacuolar degeneration occasionally exhibited mild changes, and neuronal injury was minor. The T group exhibited brain edema, with numerous neurons with vacuolar degeneration that exhibited moderate changes. The UK group exhibited partial neuronal degeneration but necrosis was rare, indicating that the brain tissues were ischemic without evident necrosis; however, irreversible necrosis may have subsequently developed if the ischemia and hypoxia did not improve. The $\mathrm{C}$ group neurons exhibited karyopyknosis, dissolution of the nuclear chromatin, marked eosin staining inside the cytoplasm and degradation of the cellular structure. The brain tissues exhibited obvious edema, with numerous neurons with vacuolar degeneration and mesh-like cells that exhibited severe alteration; the brain tissues 
were in a state of irreversible necrosis. These results suggest that the selective intra-arterial administration of combined tirofiban and urokinase possesses therapeutic value for the recovery of post-embolic brain tissues, as no new cerebral hemorrhage was observed. The present results showed that the combination of tirofiban and urokinase induced improved nerve-function recovery compared with that in the other groups. A possible mechanism may be that tirofiban improves the microcirculation of the infarcted brain tissues, inhibiting the formation of thrombus inside the microcirculatory system, and indirectly increasing microcirculatory perfusion. Furthermore, the combined treatment exhibited certain neuroprotective effects, and thus was able to enhance the restoration of neurological function.

Due to the overlapping and complementary effects of tirofiban and urokinase in the treatment of acute cerebral embolism, the combined treatment was more effective than the individual drug therapies. The present study demonstrated that an MCA injection of tirofiban prevents cerebral arterial thrombosis and induces early reperfusion, which reduces brain infarct area without inducing intracerebral bleeding. This provides a basis for the development of treatments involving the intra-arterial administration method of tirofiban. However, the current experiments used a small sample population of animals, and the universality and clinical value of the combined treatment remains unclear. Therefore, future studies are required with increased sample sizes and limited clinical application of the combined treatment, with the aim of providing an improved reference for the treatment of clinical acute cerebral infarction.

\section{References}

1. Gaillard N, Schmidt C, Costalat V, et al: Hemorrhagic risk of recent silent cerebral infarct on prethrombolysis MR imaging in acute stroke. AJNR Am J Neuroradiol 33: 227-231, 2012.

2. Sung SM, Lee TH, Cho HJ, Sol YL, Park KH, Jung DS and Kim CW: Recanalization with Wingspan stent for acute middle cerebral artery occlusion in failure or contraindication to intravenous thrombolysis: A feasibility study. AJNR Am J Neuroradiol 33: 1156-1161, 2012.

3. Kellert L, Hametner C, Rohde S, Bendszus M, Hacke W, Ringleb P and Stampfl S: Endovascular stroke therapy tirofiban is associated with risk of fatal intracerebral hemorrhage and poor outcome. Stroke 44: 1453-1455, 2013.

4. Mullen MT, Pisapia JM, Tilwa S, Messé SR and Stein SC: Systematic review of outcome after ischemic stroke due to anterior circulation occlusion treated with intravenous, intra-arterial, or combined intravenous+intra-arterial thrombolysis. Stroke 43: $2350-2355,2012$

5. Xu XQ, Zu QQ, Lu SS, et al: Use of FLAIR imaging to identify onset time of cerebral ischemia in a canine model. ANJR Am J Neuroradiol 35: 311-316, 2014.

6. Siebler M, Hennerici MG, Schneider D, et al: Safety of Tirofiban in acute ischemic Stroke: The SaTIS trial. Stroke 42: 2388-2392, 2011.

7. Georgiadis AL, Memon MZ, Shah QA, Vazquez G, Suri MF, Lakshminarayan K and Qureshi AI: Comparison of partial $(6 \mathrm{mg} / \mathrm{kg})$ versus full-dose $(9 \mathrm{mg} / \mathrm{kg})$ intravenous recombinant tissue plasminogen activator followed by endovascular treatment for acute ischemic stroke: A meta-analysis. J Neuroimaging 21: 113-120, 2011.

8. Zhang L, Zhang ZG, Zhang R, Morris D, Lu M, Coller BS and Chopp M: Adjuvant treatment with a glycoprotein IIb/IIIa receptor inhibitor increases the therapeutic window for low-dose tissue plasminogen activator administration in a rat model of embolic stroke. Circulation 107: 2837-2843, 2003.
9. Junghans U, Seitz R, Ritzl A, Wittsack HJ, Fink GR, Freund HJ and Siebler M: Ischemic brain tissue salvaged from infarction by the GP II b/III a platelet antagonist tirofiban. Neurology 58: 474-476, 2002.

10. Baik SK, Oh SJ, Park KP and Lee JH: Intra-arterial tirofiban infusion for partial recanalization with stagnant flow in hyperacute cerebral ischemic stroke. Interv Neuroradiol 17: 442-451, 2011.

11. Ottani F, La Vecchia L, De Vita M, Catapano O, Tarantino F and Galvani $\mathrm{M}$ : Comparison by meta-analysis of eptifibatide and tirofiban to abciximab in patients with ST-elevation myocardial infarction treated with primary percutaneous coronary intervention. Am J Cardiol 106: 167-174, 2010.

12. Schiariti M, Saladini A, Cuturello D, Missiroli B and Puddu PE: Long-term efficacy of high-dose tirofiban versus double-bolus eptifibatide in patients undergoing percutaneous coronary intervention. J Cardiovasc Med (Hagerstown) 12: 29-36, 2011.

13. Bederson JB, Pitts LH, Tsuji M, Nishimura MC, Davis RL and Bartkowksi H: Rat middle cerebral artery occlusion: Evaluation of the model and development of a neurologic examination. Stroke 17: 472-476, 1986.

14. Bhatia R, Hill MD, Shobha N, et al: Low rates of acute recanalization with intravenous recombinant tissue plasminogen activator in ischemic stroke: Real-world experience and a call for action. Stroke 41: 2254-2258, 2010.

15. Zangerle A, Kiechl S, Spiegel M, et al: Recanalization after thrombolysis in stroke patients: Predictors and prognostic implications. Neurology 68: 39-44, 2007.

16. Rha JH and Saver JL: The impact of recanalization on ischemic stroke outcome: A meta-analysis. Stroke 38: 967-973, 2007.

17. Mangiafico S, Cellerini M, Nencini P, Gensini G and Inzitari D: Intravenous glycoprotein IIb/IIIa inhibitor (tirofiban) followed by intra-arterial urokinase and mechanical thrombolysis in stroke. AJNR Am J Neuroradiol 26: 2595-2601, 2005.

18. Shuaib A, Yang Y, Nakada MT, Li Q and Yang T: Glycoprotein IIb/IIIa antagonist, murine 7E3 F (ab') 2 and tissue plasminogen activator in focal ischemia: Evaluation of efficacy and risk of hemorrhage with combination therapy. J Cereb Blood Flow Metab 22: 215-222, 2002.

19. Jeon JS, Sheen SH, Hwang G, Kang SH, Heo DH and Cho YJ: Intraarterial tirofiban thrombolysis for thromboembolisms during coil embolization for ruptured intracranial aneurysms. J Cerebrovasc Endovasc Neurosurg 14: 5-10, 2012.

20. Jeong HW and Jin SC: Intra-arterial infusion of a glycoprotein IIb/IIIa antagonist for the treatment of thromboembolism during coil embolization of intracranial aneurysm: A comparison of abciximab and tirofiban. AJNR Am J Neuroradiol 34: 1621-1625, 2013.

21. Chen MH, Wu SL, Lo MC, Chen WL and Wang WF: Basilar artery occlusion in a teenaged boy treated with intra-arterial thrombolysis. Acta Neurol Taiwan 19: 281-286, 2010.

22. Kwon BJ, Seo DH, Ha YS and Lee KC: Endovascular treatment of wide-necked cerebral aneurysms with an acute angle branch incorporated into the Sac: Novel methods of branch access in 8 aneurysms. Neurointervention 7: 93-101, 2012.

23. Rho MH, Kim BM, Suh SH, Kim DJ and Kim DI: Initial experience with the new double-lumen scepter balloon catheter for treatment of wide-necked aneurysms. Korean J Radiol 14: 832-840, 2013.

24. Cho YD, Lee JY, Seo JH, Kang HS, Kim JE, Jung KH and Han MH: Intra-arterial tirofiban infusion for thromboembolic complication during coil embolization of ruptured intracranial aneurysms. Eur J Radiol 81: 2833-2838, 2012.

25. Won YS, Rho MH, Kim BM, Park HJ, Kwag HJ and Chung EC: Various techniques of stent-assisted coil embolization of wide-necked or fusiform middle cerebral artery aneurysms: initial and mid-term results. J Korean Neurosurg Soc 53: 274-280, 2013.

26. Lang SH, Manning N, Armstrong N, Misso K, Allen A, Di Nisio $M$ and Kleijnen J: Treatment with tirofiban for acute coronary syndrome (ACS): A systematic review and network analysis. Curr Med Res Opin 28: 351-370, 2012.

27. Syfret DA, Mitchell P, Dowling R and Yan B: Does intra-arterial thrombolysis have a role as first-line intervention in acute ischaemic stroke. Intern Med J 41: 220-226, 2011. 